\title{
Visual Study of Multi-Dimensional Data in Remote Earth Sensing Problems
}

\author{
Boris Dobronets ${ }^{1, *}$, Olga Popova $^{1}$, and Alexey Merko ${ }^{1}$ \\ ${ }^{1}$ Institute of Space and Information Technology, Siberian Federal University, Kirenskogo 26, \\ Krasnoyarsk, 660074 Russia
}

\begin{abstract}
The article deals with the problem of multidimensional data presentation based on the application of the scientific visualization method. A method of visual-interactive animation based on dynamic representation of images in the form of $2 \mathrm{D}$ cross-sectional models is proposed. The effect of multidimensionality is achieved through the choice of $2 \mathrm{D}$ models, the variability of the directions of dynamic sections and the use of stereoscopic vision capabilities.
\end{abstract}

The task of extracting meaningful information from multidimensional data obtained using Earth remote sensing (ERS) is usually very difficult. Currently, a lot of work is being done on the use of Earth remote sensing data for solving many problems of the national economy, where it is important, first of all, to analyze the spatio-temporal changes in natural and manmade processes. Space monitoring makes it possible to obtain uniform and comparable in quality objective information of huge volumes simultaneously over vast territories, which is unattainable for any ground survey. An important feature of the incoming information is its large volume, which complicates its processing and interpretation.

It should be noted that understanding of the higher dimensions is limited. The visualization is mainly presented in three dimensions, including additional animation. Recently, approaches have been actively used for the visual presentation of multidimensional information using projections or abstractions, namely, from dimensionality reduction to visual coding and from quantitative analysis to interactive modeling.

Various techniques are currently used to visually explore multidimensional data. Among them, we note the use of perspective, the removal of hidden lines and surfaces, the use of stereographic images to represent the image of an object that deceives our visual system in the perception of space, or a three-dimensional image drawn on a clearly flat computer screen. When analyzing images in a virtual reality headset, motion parallax is applied, which helps to perceive depth, even if the display in front of us is really nothing more than an array of colored dots [1-4].

Many studies of multivariate data today use the concept of scientific visualization. Scientific visualization is based on three stages of information transformation: data transformation, visual display, viewing the transformed data. Data transformation follows analytical techniques such as dimensionality reduction, regression, clustering, feature extraction, topological analysis, data sampling, and abstraction. Visualization, as the key to most visual coding tasks, focuses on organizing information from the stage of converting data to visual presentation. Viewing the converted data follows screen-space and rendering-oriented techniques, and measures screen space to reduce artifacts and highlight important features.

\footnotetext{
*e-mail: BDobronets@yandex.ru
} 
This study focuses on the development of visual interactive animation techniques for processing, presenting and analyzing multidimensional data. It is proposed to use the method of visual-interactive modeling of spatial data to obtain the maximum possible information about the object under study. For this, an approach based on scientific visualization and visual analytics methods is used. It allows you to organize a human-machine interface that enhances the analytical abilities of a person using methods such as expanding a person's random access memory through the use of special visualization based on placing information in space in accordance with temporal relationships and organizing a controlled environment for a user to work in a space of parametric values. This organization of visual presentation and interfaces provides a person with the ability to immediately see, explore and understand huge amounts of information.

Let's consider the main methods of scientific visualization. First of all, we note the methods for reducing the dimensionality of spatial features and characteristics, which is a key factor for many visualization tasks. The main ones can be distinguished. So linear projection includes many classical methods, such as the principal component analysis (PCA), the multivariate scaling (MDS), the linear discriminate analysis (LDA) and various methods of the factor analysis [5].

Clustering is one of the most widely used methods of multivariate data analysis.Regression analysis of multivariate data is an extensive and active field of research in its own right. Researchers also use regression to summarize data. Methods like this depict data structures through wireframes and use a clustering algorithm followed by the construction of a minimal tree of cluster centroids to determine possible trends in the data. Here, regression is used as a post-processing step in algorithms to represent the totals of the extracted subsets of the data. Design management techniques are also common in which, in addition to a multidimensional input space, the user has one or more output or response variables that he wants to explore, and the results require high-quality expertise, or decision justification. We will focus on the combined application of the visualization method and regression analysis [5].

Note that visualization is primarily aimed at increasing the information content of the person solving the problem. Therefore, it is important to note that for the number of dimensions less than three, the implementation of visual data analysis is not particularly difficult, because a person has two-dimensional vision and geometric images that fit into his consciousness and representations for spaces with the number of dimensions $n \geq 3$. For multidimensional objects with a large number of dimensions, a person does not have such geometric images. Therefore, it is necessary to project into nested spaces with a standard number of dimensions. Apart from the three spatial dimensions, the only other dimension that we meet in the real world is time. Once a certain frame fusion rate is reached, there is a natural tendency to link different static images together (beta motion). Images displayed faster than about $20 \mathrm{~Hz}$ will appear continuously without flicker. During animation, processing each frame should also include restoring the render for each new time step, or perhaps reading a data file to get a new time step. One alternative to animation is to tiled the display with different frames. Note that the animation gives an overview of the object, while looking at the frames reveals small details that might otherwise have been overlooked. Therefore, ideally, we aim to provide both mechanisms for displaying multidimensional datasets [6-8].

The main feature of the considered approach to the visual-interactive representation of multidimensional data with the number of dimensions $n \geq 3$ is their dynamic representation in the form of 2D cross-sectional models. The effect of the multidimensionality is achieved through the choice of 2D models, the variability of the directions of dynamic sections and the use of stereoscopic vision capabilities. 
To improve the quality of perception, it is necessary to reduce the effect of overlapping sections. One of these methods is the ability to show not all information on the sections, but only a part. For example, apply contours using color, showing information only at the nodes of the grid. Stereo projections are used to improve perception. To reproduce isolines on sections, you can simultaneously reproduce several close sections, varying their brightness and transparency. The parameters of the method of visual-interactive animation are the distance between the planes and the speed of movement of the sections. The thickness and transparency of the isolines smoothly change depending on the plane number. The contour lines are most vividly represented in the foreground (active sections).

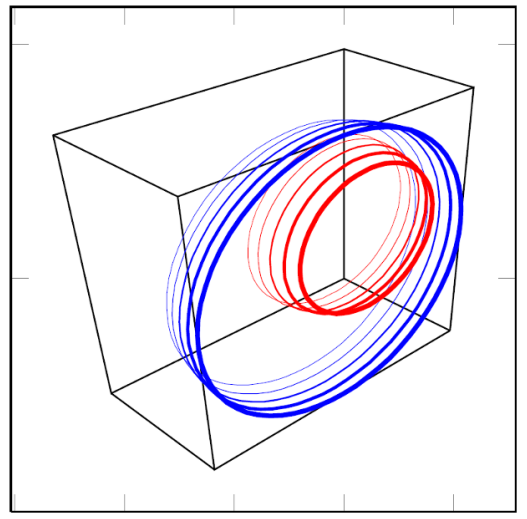

Figure 1. Isolines on sections

Figure 1 shows a fragment of the animation of the function with five sections. These sections are formed by five parallel planes. The parameters of the method of visual-interactive animation are the distance between the planes and the speed of movement of the sections. The thickness and transparency of the isolines smoothly change depending on the plane number. Isolines are most clearly shown in the foreground (active sections). The contours are shown in conventional colors, which corresponds to the value of the function. The red color corresponds to the value of the parameter $t=t_{1}$, the blue color $t=t_{2}$.

Let us dwell in more detail on the representation of an object in the form of dynamic sections. The purpose of this approach is to represent the movement of sections as an image of an object. Stereo pairs are generated for better perception. The constructed stereo image can be viewed using various equipment. Figure 2 shows a stereo image of dynamic sections.

Let's consider the most affordable. A stereoscope is an optical instrument with two eyepieces. Typically used for viewing stereo slides, it is easy to put in a laptop or communicator with an oblong high-resolution screen. A stereo display is an optical instrument by which two planar images are combined so that the observer gets a representation of the embossed object. You can use a modern TV that allows you to view stereo images through special glasses. A virtual helmet (VR HMD) is a device that displays separate images for each eye. The result is a stereo effect. The constructed stereo images were tested through a stereoscope by uploading a video file to a smartphone. The results of testing the method of visual-interactive animation showed that the most realistic picture of the presentation of three-dimensional data was obtained with dynamic reproduction of stereo images of sections with contours in conventional colors. Currently, research is underway to implement this approach for objects of dimension more than three. 

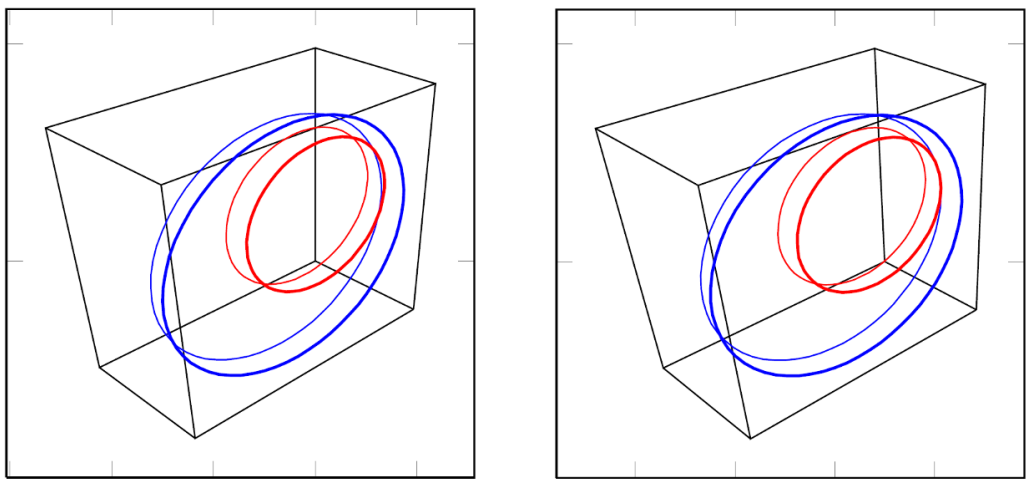

Figure 2. Stereo image of dynamic sections

The presented approach, combined with the use of numerical modeling methods, makes it possible to study remote sensing data in the tasks of studying terrain reliefs, for conducting hydrological surveys, studying agricultural land, and so on. It significantly increases the interpretability and information content of research results for various specialists.

\section{References}

[1] A.E. Bondarev, V.A. Galaktionov, V.M. Chechetkin, Computational Mathematics and Mathematical Physics 51(4), 624-636 (2011)

[2] O.P. Maslennikov, I.E. Milman, A.E. Safiulin, A.E. Bondarev, Sh.U. Nizametdinov, V.V. Pilyugin, Scientific Visualization, 6, 30-49 (2014)

[3] O.A. Popova, Informatization and communication 5 93-96 (2019)

[4] H. Wright Introduction to Scientific Visualization (London, Springer-Verlag, 2007)

[5] S. Liu, D. Maljovec, B. Wang, P.-T. Bremer, V. Pascucci, IEEE Transactions on Visualization and Computer Graphics. 23(3), 1249-1268 (2017)

[6] J. Steele (Eds.) Beautiful Visualization (OŔeilly Media, Inc., 2010)

[7] Q. Zhang, R. Segall, M. Cao, (Hershey, New York, Information Science reference, 2011)

[8] J. Dill, Expanding the Frontiers of Visual Analytics and Visualization (London: Springer-Verlag, 2012) 